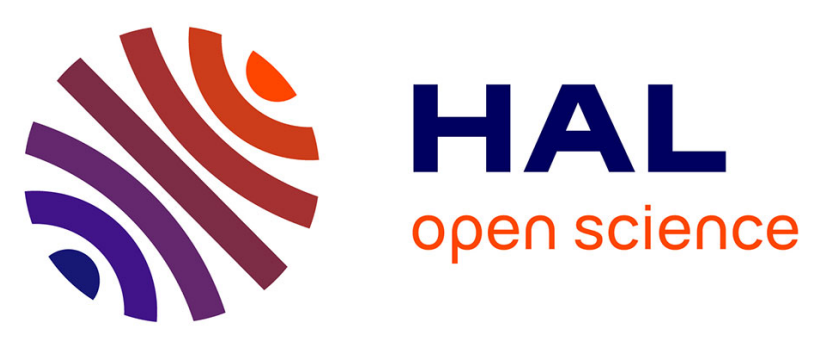

\title{
Sur les pics de frottement intérieur dus à des mécanismes faisant intervenir des volumes d'activation importants
}

J. Woirgard, M. Gerland, A. Rivière

\section{- To cite this version:}

J. Woirgard, M. Gerland, A. Rivière. Sur les pics de frottement intérieur dus à des mécanismes faisant intervenir des volumes d'activation importants. Journal de Physique Lettres, 1979, 40 (14), pp.339-340. 10.1051/jphyslet:019790040014033900 . jpa-00231639

\section{HAL Id: jpa-00231639 \\ https://hal.science/jpa-00231639}

Submitted on 1 Jan 1979

HAL is a multi-disciplinary open access archive for the deposit and dissemination of scientific research documents, whether they are published or not. The documents may come from teaching and research institutions in France or abroad, or from public or private research centers.
L'archive ouverte pluridisciplinaire HAL, est destinée au dépôt et à la diffusion de documents scientifiques de niveau recherche, publiés ou non, émanant des établissements d'enseignement et de recherche français ou étrangers, des laboratoires publics ou privés. 


\title{
Sur les pics de frottement intérieur dus à des mécanismes faisant intervenir des volumes d'activation importants
}

\author{
J. Woirgard, M. Gerland et A. Rivière \\ Laboratoire de Mécanique et de Physique des Matériaux (*), ENSMA, 86034 Poitiers Cedex, France.
}

(Reçu le 20 avril 1979, accepté le 21 mai 1979)

\begin{abstract}
Résumé. - L’influence sur les pics de frottement intérieur de la non linéarité de la vitesse des dislocations associée à des mécanismes faisant intervenir des volumes d'activation importants, a été étudiée. Il a ainsi été montré que l'expression souvent utilisée pour l'énergie d'activation, par analogie avec les essais de fluage : $E=U-\sigma v^{*}$, n'est pas applicable ( $U$ étant l'énergie à contrainte nulle, $\sigma$ la contrainte maximale et $v^{*}$ le volume d'activation).
\end{abstract}

Abstract. - The influence on internal friction peaks of non linear effects due to mechanisms with large activation volumes, is studied. It is show that the commonly used relationship : $E=U-\sigma v^{*}$, is not valid ( $E$ being the activation energy, $U$ the energy corresponding to the null stress, $\sigma$ the applied stress and $v^{*}$ the activation volume).

1. Introduction. - La sensibilité à la contrainte des effets de frottement intérieur de haute température des métaux, conduit à envisager des mécanismes élémentaires associés à des volumes d'activation très importants :

$$
v^{*}=\frac{\partial E}{\partial \sigma}
$$

$E$ étant l'énergie d'activation du mécanisme envisagé, énergie qui peut s'écrire :

$$
E=U-\sigma v^{*},
$$

en notant $U$ l'énergie à contrainte nulle.

Comme exemples de tels mécanismes on peut citer, le glissement supplémentaire d'arcs de dislocations par déplacement thermiquement activé (montée ou glissement dévié) des nœuds [1, 2], ou le glissement par montée de crans [3] de dislocations vis crantées. Dans les deux cas, le volume d'activation peut s'écrire [2] :

$$
v^{*}=\frac{b^{2} l}{2}
$$

$l$ étant la longueur moyenne des arcs dans le premier cas et la distance moyenne entre crans, dans le second.

(*) ERA CNRS n ${ }^{\circ} 123$
Pour déterminer expérimentalement $U$ et $v^{*}$ la méthode la plus directe consiste à effectuer des essais de fluage à diverses contraintes, en appliquant la relation (1). La situation est, par contre, moins simple pour les essais de frottement intérieur au cours desquels la contrainte oscille autour d'une valeur moyenne nulle.

Le but de cet article est donc de montrer que, contrairement à ce qui a souvent été avancé $[2,3,4,5]$, l'énergie d'activation apparente déduite du déplacement des pics de frottement intérieur, au cours de variations de température pour des essais isothermes, ou de variations de fréquence pour des essais anisothermes classiques, ne peut se mettre sous la forme :

$$
E=U-\sigma_{0} v^{*},
$$

cette relation n'étant applicable qu'aux essais à contrainte constante (fluage).

2. Déformation sous contrainte cyclique. - Il est maintenant admis que le frottement intérieur de haute et moyenne température des métaux est essentiellement dû au mouvement des dislocations, que ces dislocations soient ou non rassemblées en parois de polygonisation. La vitesse des segments de dislocations peut donc s'écrire :

$v=v_{l} h\left[\exp \left(-\frac{U-\sigma v^{*}}{k T}\right)-\exp \left(-\frac{U+\sigma v^{*}}{k T}\right)\right]$, 
en tenant compte de la probabilité de saut en retour. $v_{l}$ est la fréquence de vibration d'un arc de longueur $l$ et $h$ la distance parcourue à la suite d'un événement d'activation. On peut également noter cette vitesse :

$$
v=\frac{2 v_{0} b}{l} h \exp \left(-\frac{U}{k T}\right) \sinh \frac{\sigma v^{*}}{k T},
$$

où $v_{0}$ est la fréquence de Debye, $b$ le vecteur de Burgers et $T$ la température absolue.

On peut observer un maximum de frottement intérieur (pic de frottement intérieur) lorsqu'une force de rappel tend à s'opposer à la contrainte appliquée et à limiter la déformation. Cette force de rappel, que nous supposerons linéaire, peut provenir de l'action de la tension de ligne [1], ou d'interactions entre dislocations. La déformation anélastique ainsi obtenue peut alors s'écrire :

$\frac{\partial \varepsilon_{\mathrm{an}}}{\partial t}=\frac{2 v_{0} \rho b^{2} h}{l} \exp \left(-\frac{U}{k T}\right) \sinh \frac{\left(\sigma-\varepsilon_{\mathrm{an}} \delta J^{-1}\right) v^{*}}{k T}$

$\rho$ étant la densité de dislocations et $\delta J$ le défaut de compliance correspondant à la courbure maximale des arcs sous l'action de la contrainte appliquée et de la contrainte de rappel.

Pour des contraintes, ou des volumes d'activation, suffisamment faibles, on peut linéariser la relation (3) en assimilant le sinus hyperbolique à son argument :

$$
\frac{\partial \varepsilon_{\mathrm{an}}}{\partial t}=-\frac{1}{\tau} \varepsilon_{\mathrm{an}}+\frac{\delta J}{\tau} \sigma,
$$

en notant $\tau$, temps de relaxation, la quantité homogène à un temps définie par :

$$
\tau=\frac{k T}{2 v_{0}} \frac{l \delta J}{\rho b^{2} h v^{*}} \exp \frac{U}{k T} .
$$

Le spectre de frottement intérieur correspond alors à un pic de Debye dont l'amplitude et la position sont indépendantes de la contrainte.

Lorsque la contrainte ou le volume d'activation sont trop élevés pour que la linéarisation soit possible, il est possible de résoudre numériquement l'éq. (3). Dans un article récent, Esnouf et al. [3] ont proposé de négliger la probabilité de saut en retour dans la relation (2), d'introduire l'expression de la vitesse ainsi obtenue dans l'équation du mouvement (équation de la corde vibrante [6]) et de traiter l'équation non linéaire ainsi obtenue comme une équation différentielle à coefficients constants. Cette approche est incorrecte du point de vue mathématique et conduit à des paramètres d'activation, fonction de la contrainte, c'est-à-dire du temps.

Pour résoudre numériquement l'équation du mouvement, on peut introduire le paramètre sans dimension $\lambda$ :

$$
\lambda=\frac{\sigma_{0} v^{*}}{k T},
$$

la contrainte cyclique appliquée étant de la forme :

$$
\sigma=\sigma_{0} \sin \omega t
$$

On peut également poser :

$$
\begin{aligned}
& \omega t=x, \\
& \omega \tau=\alpha,
\end{aligned}
$$

et faire le changement de variable :

$$
u=\frac{\varepsilon_{\mathrm{an}}}{\sigma_{0} \delta J} .
$$

L'éq. (3) prend alors la forme simple :

$$
\frac{\partial u}{\partial x}=\frac{1}{\lambda \alpha} \sinh \lambda(\sin x-u) .
$$

Avec ces notations, le cas linéaire correspond à :

$$
\frac{\partial u}{\partial x}=\frac{1}{\alpha}(\sin x-u) ;
$$

le maximum de frottement intérieur étant obtenu pour $\alpha=1$.

Dans le cas général, il suffira donc de résoudre l'éq. (6) pour diverses valeurs du paramètre $\lambda$.

Pour que le formalisme de l'activation thermique garde un sens, il est nécessaire de ne considérer que des valeurs de $\lambda$ suffisamment faibles pour que le produit $\sigma_{0} v^{*}$ reste inférieur à l'énergie à contrainte nulle : $U$.

Dans le cas de l'aluminium les énergies d'activation déduites des essais de fluage [7] ou de frottement intérieur [8], restent inférieures à $1,5 \mathrm{eV}$, ce qui conduit à se limiter à $\lambda=15$.

Avec la condition initiale :

$$
u=0 ; t=0,
$$

la résolution numérique de l'éq. (6), par la méthode d'Euler, montre que le régime permanent est atteint au bout d'un nombre très réduit de cycles. Ce phénomène peut être vérifié sur la figure 1 , pour $\lambda=5$ et $\alpha=1$.

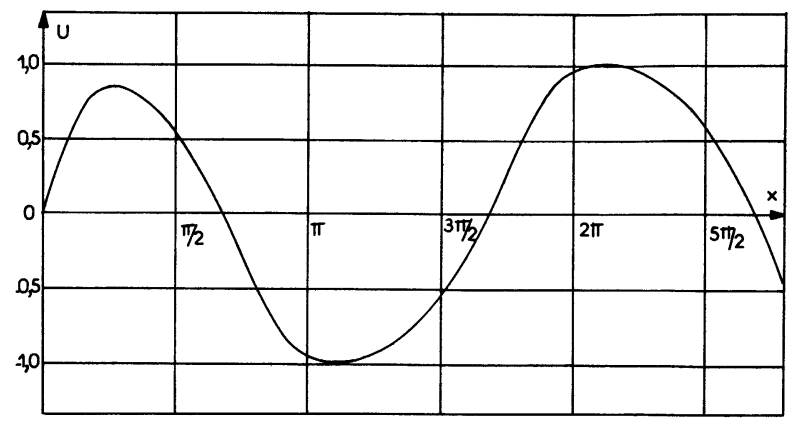

Fig. 1. - Distorsion de la déformation anélastique observée pour $\lambda=5$ et $\alpha=1$.

[Distortion of the anelastic deformation for $\lambda=5$ and $\alpha=1$.] 
Pour les fortes valeurs de $\lambda$, on note une distorsion importante qui n'est cependant sensible, compte tenu de la déformation élastique sinusoïdale superposée à la déformation anélastique, que pour des amortissements très élevés.

Avec les mesures de frottement intérieur isothermes, les seules qui conduisent à des résultats exploitables quantitativement à haute température, le frottement intérieur est obtenu, soit par mesure directe du déphasage entre la contrainte et la déformation [9], soit par mesure de la déformation au moment où la contrainte s'annule [10]. Ces deux quantités étant généralement très voisines, nous avons porté sur la figure 2 les variations avec $\alpha$ de la quantité $u(0)$.

On obtient donc un pic de frottement intérieur symétrique, d'amplitude sensiblement constante, qui tend à s'élargir et à se déplacer vers les hautes fréquences lorsque le paramètre $\lambda$ augmente.

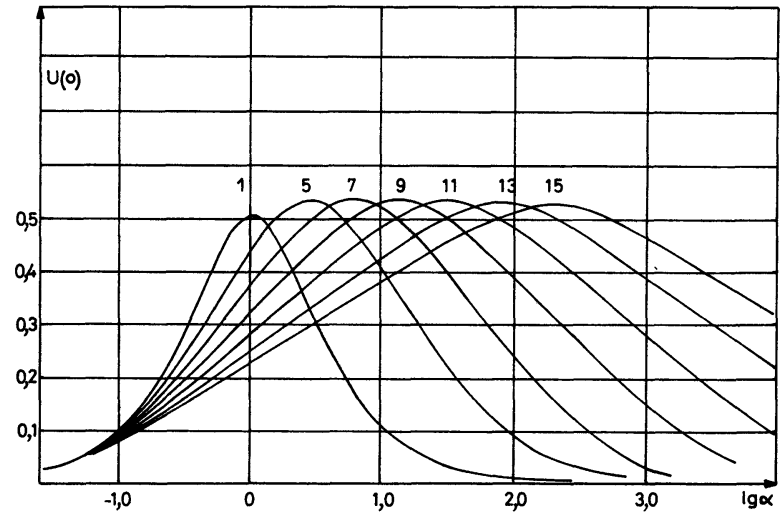

Fig. 2. - Pics de frottement intérieur obtenus pour diverses valeurs du paramètre $\lambda$.

[Internal friction peaks obtained for different values of the $\lambda$ parameter.]

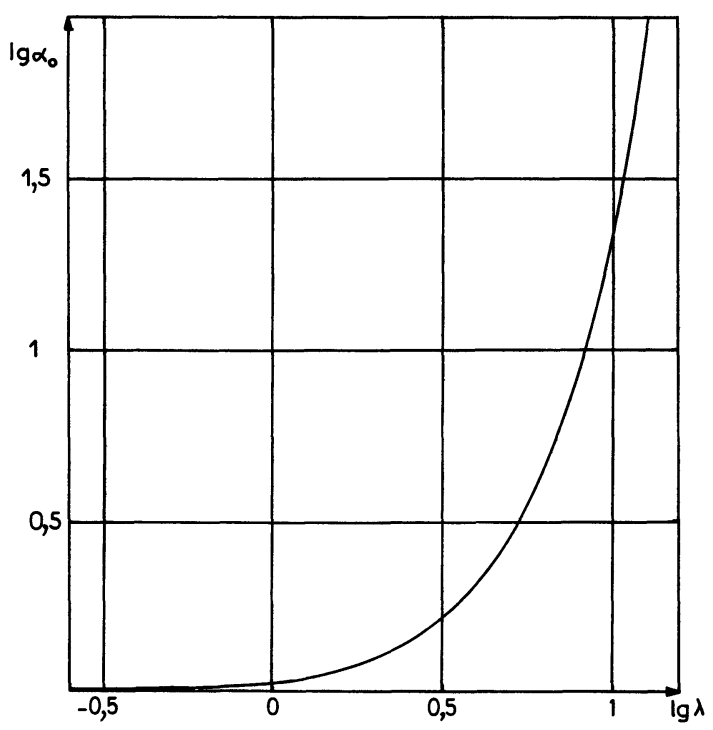

Fig. 3. - Position du pic $\left(\log \alpha_{0}\right)$ en fonction de $\log \lambda$.

[Peak position $\left(\log \alpha_{0}\right)$ versus $\log \lambda$.]
Sur la figure 3 , nous avons également porté les variations de la position du sommet du pic $\left(\log \alpha_{0}\right)$ en fonction de $\log \lambda$. Les échelles logarithmiques ont été choisies car elles permettent de déduire très simplement la valeur du volume d'activation des résultats expérimentaux.

Considérons en effet, deux pics de frottement intérieur décrits en fonction de la fréquence, à la température $T$, aux contraintes $\sigma_{1}$ et $\sigma_{2}$ et centrés respectivement sur les fréquences $\omega_{1}$ et $\omega_{2}$.

On peut calculer immédiatement :

$$
\log \frac{\alpha_{1}}{\alpha_{2}}=\log \frac{\omega_{1}}{\omega_{2}}
$$

et :

$$
\log \frac{\lambda_{1}}{\lambda_{2}}=\log \frac{\sigma_{1}}{\sigma_{2}}
$$

On en déduit immédiatement $\lambda_{1}$ et $\lambda_{2}$ (Fig. 4), c'est-à-dire $v^{*}$ :

$$
v^{*}=\frac{k T \lambda_{1}}{\sigma_{1}}=\frac{k T \lambda_{2}}{\sigma_{2}} .
$$

La relation (5) montre que l'énergie d'activation apparente déduite du déplacement du pic avec la température de mesure, à contrainte constante, peut se mettre sous la forme :

$$
E=U-\Delta E,
$$

le terme correctif $\Delta E$ dépendant de la valeur du produit $\sigma_{0} v^{*}$ et des températures considérées.

Pour deux essais effectués aux températures $T_{1}$ et $T_{2}$, il vient :

$$
\Delta E=\frac{K}{T_{1}^{-1}-T_{2}^{-1}} \log \frac{\alpha_{0}\left(\lambda_{1}\right)}{\alpha_{0}\left(\lambda_{2}\right)}
$$

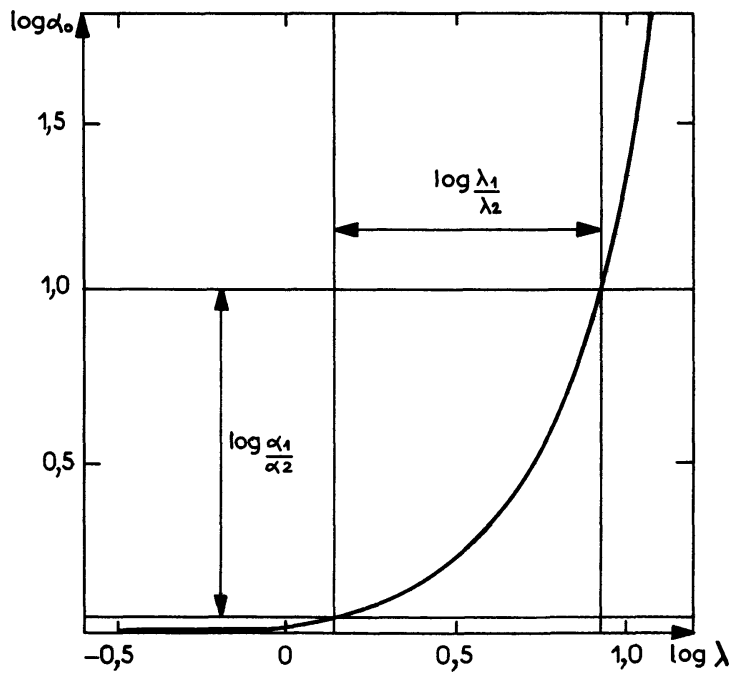

Fig. 4. - Détermination des paramètres $\lambda_{1}$ et $\lambda_{2}$ correspondant à des essais effectués aux contraintes $\sigma_{1}$ et $\sigma_{2}$.

[ $\lambda_{1}$ and $\lambda_{2}$ values obtained from two tests performed at two different stresses : $\sigma_{1}$ and $\sigma_{2}$.] 
avec

$$
\log \frac{\lambda_{1}}{\lambda_{2}}=\log \frac{T_{2}}{T_{1}}
$$

Ce terme correctif est donc généralement différent du terme $\sigma_{0} v^{*}$, déduit des essais de fluage.

Si l'on applique ce formalisme au pic observé vers $400 \mathrm{~K}$, par Esnouf et al. [3], sur l'aluminium de très haute pureté, on trouve avec $(U=0,95 \mathrm{eV})$ :

$$
v^{*} \# 3500 \mathrm{~b}^{3} \text {, }
$$

valeur nettement supérieure à celle proposée par les auteurs $\left(1500 b^{3}\right)$. En toute rigueur, l'énergie à contrainte nulle n'est certainement pas égale à $0,95 \mathrm{eV}$, mais les auteurs n'indiquant pas l'intervalle de fréquence utilisé pour déterminer l'énergie de $0,83 \mathrm{eV}$ (associée à la déformation de $2 \times 10^{-5}$ ), il n'est pas possible de déterminer, à partir de leurs résultats, la valeur exacte de $U$.

En conclusion, l'énergie d'activation apparente déduite des mesures de frottement intérieur, pour des contraintes (ou des volumes d'activation) élevées, n'est généralement pas identique à l'énergie déduite des essais de fluage effectués aux mêmes niveaux de contraintes.

\section{Bibliographie}

[1] Friedel, J., Boulanger, C. et Crussard, C., Acta Metall. 3 (1955) 380.

[2] Friedel, J., J. Physique Lett. 39 (1978) L-61.

[3] Esnouf, C., Gabbay, M. et Fantozzi, B., J. Physique Lett. 38 (1977) L-401.

[4] Smith, C. C. et LeaK, G. M., Nuovo Cimento 33 (1976) 388.

[5] Woirgard, J., Thèse D. E. (1974) Poitiers.
[6] Lenz, D. et LuCKe, K., Vth Int. Conf. on Internal Friction and Ultrasonic Attenuation in Crystalline Solids (SpringerVerlag, Berlin) II (1975) 48.

[7] DoRn, J. E., N.P.L. Conference (1956) H.M.S.O.

[8] Ke, T. S., Phys. Rev. 72 (1947) 41.

[9] Woirgard, J., Sarrazin, Y. et Chaumet, H., Rev. Sci. Instrum. 48 (1977) 10.

[10] Ke, T. S. et Ross, M., Rev. Sci. Instrum. 20 (1949) 795. 